\title{
EL ACCESO A LAS VACUNAS CONTRA EL COVID-19 Y LOS DERECHOS HUMANOS
}

\section{ACCESS TO COVID-19 VACCINES AND HUMAN RIGHTS}

\section{Barrera Vitali, Ana Carla1 - Vaggione, Gaetano²}

DOI: https://doi.org/10.37767/2591-3476(2021)06

Fecha de envío: 01.08 .2021

Fecha de aceptación: 04.10.2021

\section{RESUMEN:}

Debido a la pandemia de la COVID19, nuevas preguntas se originan sobre las vacunas, a la vez que ha evidenciado con total claridad los problemas estructurales respecto a las desigualdades e inequidades existentes entre los diferentes países. Los organismos internacionales y los propios Estados han intentado dar respuesta a través de diferentes instrumentos de la OMS, ONU y OEA, por ejemplo. Sin embargo, éstos no han podido garantizar una respuesta internacional en términos de equidad.

El presente artículo propone hacer una revisión de los principales instrumentos de organismos internacionales, observando diferencias e inequidades en la ejecución de las estrategias de vacunación en los Estados de la región. Se concluye que, en general, las respuestas de los Estados no se han adecuado a lo recomendado por organismos internacionales de salud pública. Además, se analizan las diferencias de acceso a las vacunas por parte de los Estados. Los países de mayores ingresos, con capacidad de adquirir más vacunas que los de ingresos medios y bajos, logran buenas tasas de vacunación, pero a costa de los Estados que no pueden acceder a ellas. En cuanto a las recomendaciones de los organismos internacionales, los Estados son libres de tomarlas en cuenta a la hora de diagramar políticas públicas, pero no se observa un compromiso por parte de la mayoría de los mismos de respetar estos principios propuestos en cuanto a la distribución internacional, pero sí en programas de vacunación locales.

\section{ABSTRACT}

Due to the COVID19 pandemic, new questions arise about vaccines, at the same time that the structural problems regarding the inequalities and inequities existing between the different countries have been clearly evidenced. International organizations and the States themselves

\footnotetext{
1 Estudiante avanzada de la carrera de Abogacía, Universidad Siglo 21. Integrante del equipo de Salud de Fundeps. ORCID iD: https://orcid.org/0000-00021869-3285. Correo electrónico: anacarlabarrera@fundeps.org

2 Estudiante avanzado de la carrera de Medicina, Universidad Nacional de Córdoba. Integrante del equipo de Salud de Fundeps. ORCID iD: https://orcid. org/0000-0002-8032-8908. Correo electrónico: gaetanovaggione@fundeps.org
} 
have tried to respond through different instruments of the WHO, UN and OAS, for example. However, they have not been able to guarantee an international response in terms of equity.

This article proposes to review the main instruments of international organizations, observing differences and inequities in the implementation of vaccination strategies in the States of the region. It is concluded that, in general, the response of the States has not been adequate to what is recommended by international public health organizations. In addition, the differences in access to vaccines by the States are analyzed. Higher-income countries, with the capacity to purchase more vaccines than low- and middle-income countries, achieve good vaccination rates but at the expense of states that cannot access them. Regarding the recommendations of international organizations, the States are free to take them into account when drawing up public policies, but there is no commitment on the part of the majority of them to respect these proposed principles regarding distribution. international, but in local vaccination programs.

PALABRAS CLAVE: Derechos Humanos - Derecho a la salud - Vacunación - Covid-19 Equidad.

KEY WORDS: Human rights - Right to health - Vaccination - Covid-19 - Equity.

\section{Introducción.}

Desde diciembre de 2019 el mundo se encuentra atravesando una pandemia que logró sacar a la luz grandes problemas estructurales respecto a las desigualdades e inequidades existentes entre los diferentes países. En nuestra región, la inequidad es una característica que va aumentando constantemente alimentada por las crisis económicas y las desigualdades de género y étnicas existentes. Pero, sin dudas, la principal diferencia en el acceso a la salud y, en particular, a las vacunas está marcada por la inequidad en la distribución de los ingresos que genera otras desigualdades como consecuencia y que hemos podido observar con el avance de la pandemia del Covid-19.

El derecho a la salud es un derecho fundamental y está contemplado en la Declaración Universal de los Derechos Humanos (DUDH) en el marco de la ONU, en su artículo 25 apartado 1 También, en el marco de la OEA, en el Protocolo de San Salvador, está consagrado específicamente el derecho a la salud en su Artículo 10, donde se reconoce al derecho en sí y además, estipula que son los Estados quienes deben garantizar el acceso al derecho a la salud y que para lograrlo, deben adoptar medidas a tal fin.

Actualmente, en el marco de la respuesta a la pandemia de la COVID-19, las vacunas captan toda nuestra atención. En tiempo récord, la humanidad ha conseguido desarrollar una respuesta a esta problemática, reduciendo un tiempo que suele requerir muchos años a una ambiciosa meta de 6 a 18 meses.

Esta motivación para el desarrollo científico fue lograda, e incluso superó las expectativas iniciales. Sin embargo, al abrir el espacio que conforman las diversas estrategias para abordar la pandemia, se le suma la complejidad que significa realizar campañas de vacunación masivas a nivel global. Es necesario entender, tanto a nivel de la sociedad como del funcionamiento de los sistemas de salud, que estas vacunas son herramientas que responden a un sólo enfoque de la realidad que supone que el manejo debe enfocarse desde la teoría 
del germen 3 , cuando en realidad en la actualidad es necesario incorporar esta herramienta al actual modelo de determinación social de la salud4 (Breilh, 2013: 13 - 17).

¿Pero qué sucede si los países más pobres, con economías más inestables y pocos recursos, no pueden efectivamente garantizar el derecho al acceso a la salud y el acceso a las vacunas de su población? ¿Qué sucede si los Estados que mayores recursos tienen, acaparan los insumos para hacer frente a la pandemia dejando atrás a otros países? ¿Qué sucede si esa inequidad en la distribución se convierte en una barrera global en el acceso al único elemento de lucha disponible en este momento para combatir la pandemia? ¿Qué sucede con las obligaciones internacionales que tienen los Estados para garantizar el derecho a la salud y a la vida como una comunidad internacional?

En el siguiente artículo se enumeraron las recomendaciones internacionales para las estrategias locales de vacunación. Luego, se exploraron las problemáticas en torno a dichas estrategias en la región de Latinoamérica y el Caribe. La finalidad: reflexionar sobre la efectiva aplicación por parte de los Estados de las recomendaciones planteadas a nivel universal e interamericano a la hora de ejecutar la vacunación contra la COVID19, teniendo en cuenta la inequidad presente en esta problemática .

\section{Recomendaciones internacionales \\ 1. Planes de vacunación}

Según lo recomendado por las comunidades científicas, los Estados deben tener como objetivo lograr la inmunidad de rebaño con las campañas de vacunación. Se estima que, como mínimo, para lograr esta situación se debería vacunar un 60 - 70 \% del total de la población, pudiendo llegar a ser mayor este número. A nivel global, para poder alcanzar estos números serían necesarias aproximadamente 10 - 11 mil millones de dosis, pero según relevó CEPI (2020) en una investigación, la capacidad global de producción es de 2 a 4 mil millones de dosis al año, por lo que esta meta parece un poco lejana.

A la hora de hablar del plan de vacunación, creemos que las recomendaciones de la Organización Mundial de la Salud a través de su Grupo Asesor Estratégico de Expertos en Inmunización (OMS, 2020) son la mejor opción a seguir teniendo en cuenta nuestro contexto local. Al momento de hacer nuestro análisis, Argentina se encuentra en un escenario de transmisión comunitaria y es por ello que aplicar los objetivos de esta estrategia logrará disminuir la morbimortalidad de la enfermedad, mantener los servicios esenciales y disminuir la transmisión para reducir el impacto social y económico.

Se plantean 3 etapas en base al suministro de dosis:

- Etapa 1: acceso muy limitado de dosis, para cubrir del 1 al $10 \%$ de la población. Se debe priorizar al personal sanitario en riesgo alto a muy alto de adquirir y transmitir la enfermedad; y personas adultas mayores definidas por riesgo etario (esta decisión depende de la realidad de cada país en particular).

- Etapa 2: acceso limitado de dosis, para cubrir del 11 al $20 \%$ de la población. Se debe cubrir a las personas adultas mayores no cubiertas en la etapa 1; grupos de personas con comorbilidades o estados de salud que los coloquen a un mayor riesgo

\footnotetext{
3 Teoría del germen: se entiende como esta a la teoría biomédica que supone el modelo de unicausalidad en las enfermedades, cuyo ejemplo máximo son las enfermedades infectocontagiosas. (Breilh, "La determinación social de la salud como herramienta de transformación hacia una nueva salud pública (salud colectiva)", 2013).

4 Breilh entiende como multicausalidad a la teoría biomédica que supone un modelo de múltiples factores causales que influyen a la hora de desarrollar una enfermedad.
} 
de muerte o enfermedad grave; grupos sociodemográficos a mayor riesgo de muerte o enfermedad grave; personal sanitario encargado de la entrega de dosis; personal educativo de alta prioridad.

- Etapa 3: acceso moderado de dosis, para cubrir del 21 al 50 \% de la población. Se debe priorizar al resto del personal educativo; otras personas consideradas esenciales fuera del sector sanitario y educativo; personas que están cursando una gestación; personal de salud con riesgo bajo a moderado de adquirir la enfermedad; personal involucrado en la producción de la vacuna y otro personal de laboratorio de alto riesgo; grupos sociales con riesgo elevado de adquirir o transmitir la enfermedad debido a incapacidad de mantener el distanciamiento social.

Es muy importante que los Estados compartan estas u otras indicaciones a la hora de iniciar un plan de vacunación nacional, a fin de aumentar la confianza en las instituciones y en el sistema de salud, garantizando el derecho al acceso a la información y transparencia. Actualmente, en Argentina (al día 20/7/2021) nos encontramos en un gris entre las etapas 1, 2 y 3 de la OMS, donde se están ampliando algunas indicaciones de vacunación y las poblaciones objetivo, todo esto decidido en base a la disponibilidad local de dosis, que varía ampliamente en nuestro país dependiendo de la zona geográfica y del arribo de nuevos lotes de vacunas.

\section{2. ¿Qué recomendó la OMS a los Estados?}

Dentro de la publicación de la OMS "SAGE values framework for the allocation and prioritization of COVID-19 vaccination" (2020), se describen los 6 principios y 12 objetivos que subyacen en el desarrollo de programas de vacunación para poder combatir de manera global la pandemia de la COVID-19. En nuestro análisis sobre los programas de vacunación que se ejecutan a nivel local, regional y global encontramos múltiples fallas a la hora de cumplir estas recomendaciones.

En este documento, se establece que:

(...) las decisiones sobre cómo implementar una limitada vacunación para la COVID-19 no debe basarse únicamente en consideraciones de la salud pública. Tampoco deberían ser impulsados por consideraciones económicas por sí solas, a pesar de que el impacto de esta pandemia en las economías de las naciones y la seguridad financiera de las familias ha sido para muchos devastador. (OMS, 2020, pág. 4)

Además, describe cómo esta herramienta funciona tanto como guía para las personas encargadas de diseñar y ejecutar las políticas públicas y sanitarias, y también como herramientas de accountability.

Con el objetivo de realizar una lectura crítica de la realidad de los planes de vacunación a nivel mundial, es que destacamos estos principios y objetivos dentro del documento a fin de analizar si los Estados cumplen o no con las recomendaciones de la Organización Mundial de la Salud.

El principio fundamental es el que hace referencia al bienestar humano ("human wellbeing") y sus objetivos tales como la reducción de las muertes y la carga de la enfermedad por la pandemia de la COVID-19; la disminución de la disrupción social y económica mediante contención de la transmisión; lograr reducir la enfermedad severa y la muerte, o una combinación de estas estrategias y proteger el continuo funcionamiento de los servicios esenciales, incluyendo los servicios de salud. 
El segundo principio a destacar, es el de respeto igualitario ("equal respect"), cuya finalidad es adoptar los intereses de todos los individuos y grupos con igual consideración mientras las decisiones de asignación y de establecimiento de prioridades están siendo tomadas y ejecutadas. Además, también busca ofrecer una oportunidad significativa de ser vacunados a todos los individuos y grupos que califican bajo criterios de priorización.

Destacamos también el Principio de equidad global ("global equity"), que plantea como objetivos asegurar que la asignación de vacunas tenga en cuenta los riesgos epidémicos especiales y las necesidades de todos los países, particularmente países de ingresos bajos y medios. También busca que todos los países se comprometan a cumplir las necesidades de las personas que viven en países que no pueden garantizar las vacunas para su población, especialmente aquellos de ingresos bajos y medios. Junto con este Principio de equidad global, se encuentra el Principio de equidad nacional ("national equity"), cuyos objetivos son asegurar la priorización dentro de los países para tener en cuenta las vulnerabilidades, riesgos y necesidades de grupos que, por causas de tipo sociales, geográficas o biomédicas, se encuentren propensos a sufrir mayores riesgos de carga de la enfermedad pandémica. Además, tiene como objetivo desarrollar los sistemas de distribución de inmunizaciones y la infraestructura necesaria para asegurar el acceso a las vacunas a poblaciones prioritarias y tomar acciones proactivas para asegurar igual acceso a cualquiera que califique en un grupo prioritario, especialmente las poblaciones socialmente vulnerables.

\section{La Comisión Interamericana de Derechos Humanos y la vacunación para la COVID-19}

El 6 de abril de 2021, la Comisión Interamericana de Derechos Humanos (en adelante CIDH) adoptó la Resolución nro. 01/2021 donde se aboca a tratar la situación de las vacunas en el marco de las obligaciones interamericanas de derechos humanos. En dicha resolución, la $\mathrm{CIDH}$, acompaña los pronunciamientos realizados por el Comité DESC y también al Ilamamiento que el Consejo de Derechos Humanos de la ONU dispuso en la resolución A/HRC/46/L.25/Rev.1 del 17 de marzo de 2021, donde se insta a asegurar el acceso a las vacunas de forma equitativa, asequible, oportuna y universal para que todos los países puedan enfrentarse a la pandemia.

La CIDH busca lograr su objetivo de que todos los países miembros de la OEA asuman sus responsabilidades internacionales respecto a la vacunación y pueda garantizarse efectivamente el derecho a la salud y la vida, y para eso realiza una serie de recomendaciones que se sustentan en los principios de igualdad y no discriminación, dignidad humana, consentimiento informado, transparencia, acceso a la información, cooperación y solidaridad internacional. Principalmente, sostiene que las vacunas deben considerarse un bien de salud pública y que el acceso a las mismas debería ser libre y son los Estados quienes tienen la obligación de garantizar de modo inmediato el acceso universal y equitativo de las mismas a todas las poblaciones. Así, dispone de 6 recomendaciones para los Estados miembros:

1. Acceso a las vacunas, bienes y servicios de salud en atención al principio de igualdad y no discriminación, asegurando la distribución de las vacunas y su acceso equitativo y universal, adoptando medidas que lo garanticen com la implementación de planes nacionales de vacunación.

2. Distribución y priorización de dosis de vacunas para aquellas personas con mayores riesgos de contagio y para quienes experimentan un riesgo mayor para enfrentar a la pandemia. Para establecer los criterios, sugiere utilizar los parámetros estableci- 
dos por la SAGE de la OMS (2020).

3. Difusión activa de información adecuada y suficiente sobre las vacunas y contrarrestar la desinformación existente sobre las mismas. Señala que es necesario que los Estados fortalezcan la seguridad de las instituciones de salud pública y el conocimiento de base científica.

4. Garantizar el derecho al consentimiento previo, libre e informado de las personas que reciben las vacunas para que puedan acceder a información de manera oportuna, completa, comprensible, clara, sin tecnicismos, fidedigna, culturalmente apropiada y que tome en cuenta las particularidades y necesidades específicas de quien la recibe.

5. Derecho de acceso a la información, transparencia y combate contra la corrupción para que toda la población pueda acceder a los datos relativos a la compra de vacunas, el estado de los planes de vacunación, dosis disponibles y demás datos vinculados a la vacunación en el país.

6. Por último, insta a que los Estados garanticen que todas las decisiones relativas al desarrollo, la utilización y la distribución de vacunas por parte de las empresas tengan en cuenta los principios transversales de derechos humanos tales como la transparencia, la información, la igualdad y no discriminación, la rendición de cuentas y el respeto a la dignidad humana.

\section{Desigualdades de la pandemia en el contexto internacional}

Es conocido el impacto de la pandemia en todas las economías mundiales, pero hay que hacer un análisis crítico de la forma en que impactó más específicamente dentro de cada Estado en particular. Ya las Naciones Unidas en el Informe del Secretario General sobre el "Progreso realizado para lograr los Objetivos del Desarrollo Sostenible", del Consejo Económico y Social (ONU, 2020), estima que los países vulnerables (ya sea por motivos económicos, geopolíticos o por crisis humanitarias) tendrán a largo plazo consecuencias más complejas de resolver, ya sea por la fragilidad de sus sistemas sanitarios, la poca cobertura de protección social, las limitaciones financieras y económicas o por su dependencia del comercio internacional.

A su vez, según los Indicadores de los ODS de la División Estadística del Departamento de Asuntos Económicos y Sociales de las Naciones Unidas (ONU, n.d.) estos datos se repiten, no sólo afectando a la relación de Estados entre sí, sino también afectando a la realidad interna de cada Estado, aumentando la brecha de distribución de la riqueza entre los sectores sociales más privilegiados y quienes se encuentran en situación de vulnerabilidad. Apoyando esto, un Comunicado de Prensa del Banco Mundial (2020) refiere que el 82\% del total de todas las personas que se encontrarán bajo el umbral de la pobreza extrema se ubicarán en países de ingresos bajos y medios.

Hay que destacar que este impacto se maximiza de manera especialmente cruenta en los países de bajos ingresos. Según una publicación de Oxfam (2021) sobre la materia, los países que tenían un nivel de deuda alto o potencialmente podían tenerlo, se ha registrado una reducción del $20 \%$ en remesas y una caída del $25 \%$ en inversión extranjera y en comercio, que sumada a una caída de precios y a una fuga de capital ha obligado a estos Estados a posponer sus compromisos de deuda, pero posponiendo sólo una pequeña fracción de las obligaciones a cumplir. También se estima que los ingresos fiscales han caído, con una caída del 2,6\% del PBI en África subsahariana del 2019 al 2020. Se estima, además, que en América Latina se ha perdido un 59\% del total del gasto público de salud en la región. 
Estas inequidades no sólo se reflejan en el impacto económico de la pandemia, sino que también se traslada a la posibilidad de fortalecer el sector salud por parte de los Estados. Según un estudio realizado por el World Inequality Lab (2021), los países de ingresos medios y bajos no han podido realizar gastos en salud de más del $6 \%$ de su PBI, mientras que los de altos ingresos en promedio han podido destinar hasta el 12\%, el doble. Esta diferencia también se observó en las medidas para atacar la pandemia, donde los países de ingresos bajos y medios basaron sus estrategias para enfrentar la pandemia en medidas costo-efectivas (utilizando controles de temperatura, desinfección de manos, distanciamiento social, cierre de sectores y cuarentenas) mientras que los países de altos ingresos pudieron realizar medidas de mayor tecnología y costo (testeos masivos, campañas de información, distanciamiento social y cuarentenas). Lo importante de estas medidas es la capacidad de poder mantener por más tiempo las medidas de control que implican el distanciamiento social y la caída de la actividad económica, ya que los países de altos ingresos tuvieron la posibilidad de realizar un apoyo económico a los negocios y personas que más fueron golpeadas, mientras que los países de ingresos bajos y medios no cuentan con esta espalda financiera, por lo que volver a realizar cuarentenas restringidas se vuelve una herramienta no aplicable en futuros brotes.

Teniendo en cuenta que estas opciones no se vuelven viables, y que el lograr vacunar a un porcentaje determinado de la población implicaría poder retorna de manera progresiva a la actividad económica normal, emerge la situación de que las vacunas se convierten así en un bien escaso preciado que entra en jaque con la idea de equidad internacional para lograr que todos los Estados puedan hacer frente a la COVID19.

\section{Inequidad internacional}

Ahora bien, ¿qué sucede con las campañas de vacunación a una escala global? Lo primero a destacar, es que más allá de los distintos tipos de vacunas (que se analizarán más adelante), los diversos Estados han afrontado sus planes locales de vacunación de manera muy dispar.

Un fenómeno llamativo, que podría ocasionar consecuencias dramáticas, es la brecha de acceso ("access gap") a las vacunas entre los países de altos ingresos y los de bajos ingresos. Según los informes realizados por la iniciativa "Launch and Scale Speedometer" del Duke Global Health Innovation Center (2021), los países de altos ingresos se han asegurado 4.713.277.500 dosis, mientras que los de ingresos medio bajos y bajos se han asegurado 732.028 .000 y 70.200 .000 dosis respectivamente. La iniciativa COVAX ${ }^{5}$ a su vez ha conseguido 1.120.000.000 dosis.

A la hora de analizar la cantidad de dosis adjudicadas por habitante, esta brecha de acceso entre los Estados se vuelve abrumadora. Países de altos ingresos como Canadá, el Reino Unido y Nueva Zelanda se han asegurado 8,67; 7,29 y 6,57 dosis por habitante, respectivamente, mientras que la gran mayoría de los países de ingresos moderados y moderados - bajos no cuentan con la suficiente cantidad de dosis para vacunar a toda su población (a pesar de algunas excepciones a la hora de los contratos de dosis). Por ejemplo, Venezuela a la fecha de este informe (30/07/2021) ha podido asegurar por contrato 22 millones de dosis (10 millones de Gamaleya y 12 millones del Center for Genetic Engineering and Biotechnology) para una población total de 27 millones de personas.

5 Entiéndase que por COVAX nos referimos al mecanismo coordinado por Gavi, la Vaccine Alliance, la CEPI y la OMS que tiene como objetivo lograr la igualdad en la distribución de vacunas para la COVID19. 
Esta inequidad no solo prolonga los tiempos de espera y restringe la capacidad de recuperación económica de los países de ingresos moderados y moderados - bajos (países que también han recibido el mayor impacto económico de esta pandemia), sino que también afecta en sus tasas de mortalidad y de supervivencia.

Chinazzi, Davis, Dean, Mu, Pastore y Xiong (2020) analizaron (mediante un modelo global de transmisión de metapoblaciones) 2 escenarios posibles (cooperativo vs no cooperati$v^{6}{ }^{6}$ ) de distribución de 3 mil millones de dosis. Con una vacuna con una eficacia del $80 \%$, se estimó que en el escenario no cooperativo se evitaría aproximadamente un 33\% de muertes, mientras que el escenario cooperativo estimó evitar un $61 \%$ de muertes.

Es importante destacar que, si bien se pueden evitar casi el doble de muertes con una estrategia cooperativa, la estrategia no cooperativa lograría una ganancia modesta en cuanto a muertes evitadas en los países de altos ingresos. Por ejemplo, en Europa Occidental se estimó una diferencia del 19\% de muertes evitadas al pasar de un escenario cooperativo a uno no cooperativo; mientras que en Norteamérica la misma fue del $14 \%$. En contraste con las diferencias obtenidas en otras regiones, en África Occidental se observó una diferencia del $80 \%$ de muertes evitadas al pasar de un escenario no cooperativo a uno cooperativo (de 13\% al 93\%); mientras que en el Sudeste Asiático se observó una diferencia del $57 \%$ (de $5 \%$ al $62 \%$ ) de muertes evitadas.

Para responder a esta problemática de manera global y colaborativa, se creó la iniciativa COVAX. Esta plataforma es parte del ACT Accelerator ("Access to COVID-19 Tools") y está coordinada por Gavi, la Vaccine Alliance, la CEPI y la OMS. Cualquier país puede participar de la misma, y se garantiza que contarán con un igual acceso al desarrollo de las vacunas. Se espera que se desarrollen 2 mil millones de dosis para fines del 2021. Es así que esta iniciativa busca suplir la demanda de tanto países de altos ingresos como de bajos ingresos, y cumplimentar sus necesidades de dosis de manera igualitaria hasta cubrir el $20 \%$ de la población de cada uno (Berkley, 2020).

Sin embargo, la brecha de acceso a las vacunas no afecta sólo a la cantidad de vacunas que pueden adquirirse por contrato. Se observan brechas significativas entre los países de ingresos altos y los de ingresos medios y medios - bajos en cuanto al precio de dosis por contrato estatal, lo cual aumentaría la brecha de acceso y obligaría a los Estados a explorar nuevas formas de adquirir las dosis necesarias para lograr la inmunidad de rebaño.

Las descripciones de las vacunas aquí mencionadas forman parte del estudio realizado por Funk, Laferriere y Ardakani (2021). El compuesto activo de la vacuna de Pfizer-Biotech y Moderna (BNT162b1, con la diferencia de cantidad de dosis y días entre las 2 colocaciones) se destaca por ser de los primeros en la "carrera" para desarrollar campañas de vacunación para la COVID-19, y por alcanzar niveles de eficacia del 94-95\%. Por otro lado, las vacunas que utilizan vectores virales cuentan con eficacias muy variables, con la vacuna de Astra Zeneca Oxford (AZ/Ox) / Covishield del Serum Institute of India con una eficacia del 62,1\% ; mientras que la vacuna de Gamaleya tiene una eficacia del 91,6\%; J\& o Janssen cuenta por el contrario con una efectividad del 66\%. Por último, las vacunas in-

6 En este estudio se entiende por escenario cooperativo en dicho estudio a una distribución por país proporcional a la población total de cada uno, mientras que en el escenario no cooperativo el $66 \%$ de las dosis son adquiridas por un pequeño grupo de países de altos ingresos, y el porcentaje sobrante se distribuye de manera equitativa entre el resto de los países. 
activadas como la de Sinopharma (Beijing) tiene una efectividad registrada del 79,3\%; y la de Bharat Biotech tiene una eficacia estimada mayor al 50\% al momento de la realización de la revisión sistemática citada.

Esta revisión también hace una valoración integral de todos los tipos de vacunas, y las organiza teniendo en cuenta su seguridad/reactividad, eficacia, régimen de dosis, estabilidad del producto/almacenamiento/cadena de distribución y precio objetivo/accesibilidad.

Teniendo en cuenta estas variables, las vacunas que mejor cubren los estándares son las que utilizan RNA (es decir, las de Pfizer-Biotech y Moderna), pero en nuestra región tienen dificultades con las cadenas de distribución relacionadas principalmente con la falta de infraestructura regional para poder mantener dichas cadenas, especialmente la de frío. En cuanto a costos, según la UNICEF Supply Division (2021), la vacuna de PfizerBiotech se comercializa desde 6,75 dólares en África, llegando a costar 18,90 dólares en Europa y hasta 19,50 en Estados Unidos. En el mismo tipo de vacunas, la de Moderna inicia su precio en 15,00 dólares para los Estados Unidos hasta llegar a los 32,00 o 37,00 dólares para países de altos ingresos.

Por otra parte, la vacuna de AstraZeneca/Ox cuesta desde los 2,06 dólares a India, 3,00 dólares para la Unión Africana y COVAX AMC, 4,00 dólares para Estados Unidos y Latinoamérica, 5,30 dólares para India hasta llegar a los 13,27 dólares para el mercado privado de Bangladesh. La vacuna Gamaleya Sputnik V se vende desde los 3,00 dólares en LATAM, pasando por el precio de 10,00 dólares para venta global, para luego llegar a valer 19,90 en Hungría, y hasta 27,15 en el mercado privado de Pakistán. Por último, en este tipo de vacunas, la producida por J\&J (Jenssen) se vende desde 8,50 dólares en Europa hasta los 10,00 dólares en Estados Unidos y África.

En contraste, la vacuna inactivada de Sinopharm (del Beijing Institute of Biological Products) va desde los 18,60 dólares en Senegal, pasando a los 29,75 en China, los 36,00 dólares para Hungría hasta llegar a los 40,00 dólares por dosis para Argentina. Y por último, la vacuna inactivada de Bharat Biotech de India (Covishield) se comercializa desde 4,00 dólares en India hasta 35,00 en el mercado privado de Nepal.

Ahora, entre tanta información y variables, cabe preguntarse cuál vacuna es la "mejor". Según los "Target Product Profiles for COVID-19 Vaccines" de la Organización Mundial de la Salud (2020), se establecen las características que deberían cumplir las vacunas para poder utilizarse en el marco de la pandemia que estamos transitando, y se plantean características "óptimas" y "críticas o mínimas". En cuanto a la eficacia, se considera óptimo que cuente con una eficacia mayor al 70\% en estudios de población (lo cual debe determinarse con un punto de corte para prevención de enfermedad, de enfermedad severa y reducción de la tasa de transmisión). Sin embargo, como criterios mínimos o críticos este porcentaje de eficacia disminuye hasta lograr superar aproximadamente el 50\%. Si analizamos todas las vacunas disponibles en nuestro territorio, es posible observar que todas cumplen con este imperativo mínimo a la hora de utilizarse. Por lo tanto, a la hora de definir que nos inclina a elegir entre una y otra vacuna, lo más decisivo sería la accesibilidad y capacidad de abastecerse para vacunar en el menor período de tiempo a la mayor parte de la población, para lograr tasas de cobertura mayores a las indicadas en este documento, a fin de disminuir la tasa de replicabilidad del Sars-CoV-2 a un valor menor a 1. El precio es también un factor importante, ya que en última instancia puede 
definir la postura de negociación de un Estado con una farmacéutica, ya que precios demasiado elevados por dosis pueden ser una barrera al acceso. En resumidas cuentas, la mejor vacuna es la que se encuentra disponible para ser administrada.

\section{Situación regional: ¿cómo avanza la vacunación?}

En nuestra región observamos diferencias significativas en el ritmo de vacunación entre los países de altos ingresos y de ingresos medios y medios-bajos. Se pueden observar las diferencias de impacto de las campañas de vacunación, que va de la mano de la inequidad ya descrita entre los Estados en el acceso a las vacunas. Se observa que los Estados con la mayor cantidad de esquemas completos corresponden a países de ingresos altos en relación a los de ingresos medio-bajo, potencialmente aumentando la brecha de impacto de la pandemia sobre los mismos.

\begin{tabular}{|c|c|c|c|c|c|c|}
\hline $\begin{array}{l}\text { País ( nivel } \\
\text { de } \\
\text { ingresos) }\end{array}$ & $\begin{array}{l}\text { Población } \\
\text { total }\end{array}$ & $\begin{array}{l}\text { 1eras dosis } \\
\text { administra } \\
\text { das }\end{array}$ & $\begin{array}{l}\text { Esquemas } \\
\text { completos } \\
\text { totales }\end{array}$ & $\begin{array}{l}\text { Dosis } \\
\text { administra } \\
\text { das cada } \\
100 \\
\text { personas }\end{array}$ & $\begin{array}{l}\text { Esquemas } \\
\text { completos } \\
\text { cada } 100 \\
\text { personas }\end{array}$ & $\begin{array}{l}\text { Porcentaje } \\
\text { de } \\
\text { población } \\
\text { cubierta } \\
\text { por } \\
\text { acuerdos } \\
\text { bilaterales } \\
\text { y } \\
\text { multilatera } \\
\text { les } \\
\text { (incluyendo } \\
\text { COVAX) }\end{array}$ \\
\hline $\begin{array}{l}\text { Argentina } \\
\text { (país de } \\
\text { ingresos } \\
\text { medio - } \\
\text { alto) }\end{array}$ & 45.605 .823 & 22.876 .517 & 5.797 .847 & 62,87 & 12,71 & $54,34 \%$ \\
\hline $\begin{array}{l}\text { Brasil ( país } \\
\text { de ingresos } \\
\text { medio - } \\
\text { alto) }\end{array}$ & $\begin{array}{l}213.993 .44 \\
1\end{array}$ & 88.891 .440 & 34.400 .170 & 57,61 & 16,08 & $151,03 \%$ \\
\hline $\begin{array}{l}\text { Chile ( país } \\
\text { de ingresos } \\
\text { altos) }\end{array}$ & 19.212 .362 & 12.622 .222 & 12.091 .227 & 128,6 & 62,93 & $222,77 \%$ \\
\hline $\begin{array}{l}\text { Colombia } \\
\text { (país de } \\
\text { ingresos } \\
\text { medio - } \\
\text { alto) }\end{array}$ & 51.265 .841 & 13.646 .720 & 10.889 .063 & 47,860 & 21,24 & $54,13 \%$ \\
\hline
\end{tabular}

7. Esta tabla se conformó con la información otorgada por la "COVID-19 Vaccination in the Americas" de la Organización Panamericana de la Salud (2020) y por la "COVID-19 Vaccine Market Dashboard" de UNICEF, para actualizarse a la fecha del 30/07/2021. 


\begin{tabular}{|l|l|l|l|l|l|l|}
\hline $\begin{array}{l}\text { Perú ( país } \\
\text { de ingresos } \\
\text { medio - } \\
\text { alto) }\end{array}$ & 33.359 .416 & 7.108 .859 & 4.217 .276 & 33,95 & 12,64 & $109,48 \%$ \\
\hline $\begin{array}{l}\text { Bolivia } \\
\text { (país de } \\
\text { ingresos } \\
\text { medio - } \\
\text { bajo) }\end{array}$ & 11.832 .936 & 2.742 .827 & 1.144 .931 & 32,86 & 9,68 & $155,08 \%$ \\
\hline $\begin{array}{l}\text { Paraguay } \\
\text { (país de } \\
\text { ingresos } \\
\text { medio - } \\
\text { alto) }\end{array}$ & 7.219 .641 & 1.769 .429 & 247.305 & 27,93 & 3,43 & $30,47 \%$ \\
\hline $\begin{array}{l}\text { Uruguay } \\
\text { (país de } \\
\text { ingresos } \\
\text { altos) }\end{array}$ & 3.485 .152 & 2.510 .872 & 2.112 .404 & 132,7 & 60,61 & $53,80 \%$ \\
\hline
\end{tabular}

\section{Conclusiones}

Con la actual crisis ocasionada por la pandemia del Covid-19, las situaciones de desigualdades estructurales y las inequidades en la distribución de los recursos se han incrementado y se han evidenciado. Ante un problema global que requiere respuestas multilaterales y coordinadas, es imperativo que, para esta actual pandemia y futuras, los Estados asuman sus responsabilidades y obligaciones internacionales para garantizar y asegurar el acceso a los derechos fundamentales, y en este caso, al derecho a salud y el acceso a las vacunas de forma equitativa, asequible, oportuna y universal para que todos los países puedan enfrentarse a las futuras enfermedades en pie de igualdad.

Resaltando la importancia de lo expuesto por la CIDH (2021) las vacunas deben considerarse un bien de salud pública y que el acceso a las mismas debería ser libre y son los Estados quienes tienen la obligación de garantizar de modo inmediato el acceso universal y equitativo de las mismas a todas las poblaciones.

Es importante destacar el rol que tiene la distribución de vacunas en la principal estrategia que hoy en día existe para enfrentar la pandemia. La acumulación de dosis en los países de mayores ingresos produce un desabastecimiento de vacunas en los países de menores recursos.

El no poder mantener un ritmo de vacunación constante no solamente implica un aumento de mortalidad y transmisibilidad de la población, sino que también son campos de cultivo para el desarrollo de nuevas cepas y variantes, lo cual implica el riesgo de sufrir nuevos brotes epidemiológicos, con el potencial riesgo de una cepa que modifique los componentes antigénicos presentes en las vacunas actuales. Esto sería catastrófico, ya que nuestras vacunas serían (teóricamente) no efectivas contra una cepa de estas características. 
Lo más decisivo en cuanto a la problemática sería la accesibilidad y capacidad de abastecerse para vacunar en el menor período de tiempo a la mayor parte de la población, para lograr tasas de cobertura mayores a las indicadas en este documento, a fin de disminuir la tasa de replicabilidad del Sars-CoV-2 a un valor menor a 1.

En resumidas cuentas, la mejor vacuna es la que se encuentra disponible para ser administrada. En este sentido, la falta de cumplimiento de las recomendaciones de organismos internacionales aumentaría la inequidad en los planes de vacunación, generando diferencias entre los países de altos ingresos frente a los de ingresos medios y medios-bajos.

\section{REFERENCIAS BIBLIOGRÁFICAS}

- Banco Mundial. (2021). Debido a la pandemia de COVID-19, el número de personas que viven en la pobreza extrema habrá aumentado en 150 millones para 2021. Comunicado de prensa del Banco Mundial, 7-10-2021. Grupo Banco Mundial. Disponible en: https:// www.bancomundial.org/es/news/press-release/2020/10/07/covid-19-to-add-as-manyas-150-million-extreme-poor-by-2021

- Berkley, S. (2020). COVAX explained. Gavi.Org [blog], 3-9-21. Disponible en: https://www. gavi.org/vaccineswork/covax-explained

- Breilh, Jaime. (2013). La determinación social de la salud como herramienta de transformación hacia una nueva salud pública (salud colectiva). Revista Facultad Nacional de Salud Pública, 31( ),13-27.[fecha de Consulta 19 de Julio de 2021]. ISSN: 0120-386X. Disponible en: https://www.redalyc.org/articulo.oa?id=12028727002

- CEPI Sustainable Manufacturing Team. (2020). CEPI survey assesses potential COVID-19 vaccine manufacturing capacity. Cepi.Net [blog], 5-8-21. Disponible en: https://cepi.net/ news_cepi/cepi-survey-assesses-potential-covid-19-vaccine-manufacturing-capacity/

- Chinazzi, M., Davis, J. T., Dean, N. E., Mu, K., Pastore, P. A., \& Xiong, X. (2020). Estimating the effect of cooperative versus uncooperative strategies of COVID-19 vaccine allocation: a modeling study. Network Science Institute, Northeastern University. Disponible en: https://www.mobs-lab.org/uploads/6/7/8/7/6787877/global_vax.pdf

- Comisión Interamericana de Derechos Humanos [CIDH]. (2021) Resolución 01/20221:Las vacunas contra el Covid-19 en el marco de las obligaciones interamericanas de los derechos humanos - Disponible en https://www.oas.org/es/cidh/decisiones/pdf/Resolucion1-21-es.pdf

- Duke University. (2021). Vaccine Purchases. Launchandscalefaster.Org [blog], 1-112021. Disponible en: https://launchandscalefaster.org/covid-19/vaccinepurchases

- Funk, C. D., Laferrière, C. y Ardakani, A. (2021). Target Product Profile Analysis of COVID-19 Vaccines in Phase III Clinical Trials and Beyond: An Early 2021 Perspective. Viruses, 13(3), 418. Disponible en: https://doi.org/10.3390/v13030418

- Hopenhayn, Martín. (2006) - Reunión de expertos sobre población, desigualdades y derechos humanos: Desigualdades Sociales y derechos humanos: Hacia un pacto de protección social (CELADE, División de la Población de la CEPAL). Santiago de Chile: CEPAL. Disponible en https://www.cepal.org/sites/default/files/events/files/hopenhaynm.pdf - Organización de los Estados Americanos. (1988) - Protocolo Adicional a la Convención Americana de Derechos Económicos, Sociales y Culturales "Protocolo de San Salvador". Wash- 
ington: OEA. Disponible en http://www.oas.org/es/cidh/mandato/Basicos/sansalvador.asp - Organización de las Naciones Unidas. (2021). Resolución del Consejo de Derechos Humanos, Asamblea General de las Naciones Unidas; A/HRC/46/L.25/Rev.1 - Asegurar el acceso equitativo, asequible, oportuno y universal de todos los países a las vacunas para hacer frente a la pandemia de enfermedad por coronavirus (COVID-19) Ginebra: ONU . Disponible en: https://undocs.org/es/A/HRC/46/L.25/Rev.1

- Organización de las Naciones Unidas. (2020). Progresos realizados para lograr los Objetivos del Desarrollo Sostenible. Informe del Secretario General, (Informe del Secretario General, Consejo Económico y Social, Asamblea General de las Naciones Unidas; E/2020/57). Nueva York: ONU . Disponible en: https://unstats.un.org/sdgs/files/report/2020/secretary-general-sdg-report-2020--ES.pdf

- Organización de las Naciones Unidas. (2020). Sustainable Development Goals Indicators. Unstats.Un.Org [blog], 2020. Disponible en: https://unstats.un.org/sdgs/report/2020/goal-10/ - Organización Panamericana de la Salud. (2021). COVID-19 vaccine doses administered in the Americas. Ais.Paho.Org [blog], 1-10-2021. Disponible en: https://ais.paho.org/imm/ IM_DosisAdmin-Vacunacion.asp

- Organización de las Naciones Unidas. (1948). La Declaración Universal de los Derechos Humanos (Declaración de la Asamblea General, Naciones Unidas). Nueva York: ONU. Disponible en https://www.un.org/es/about-us/universal-declaration-of-human-rights

- Oxfam. (2021). El Virus de la Desigualdad: Cómo recomponer un mundo devastado por el coronavirus a través de una economía equitativa, justa y sostenible (Oxfam GB, Oxfam Internacional). Oxford: Oxfam. Disponible en: DOI: 10.21201/2020.6409

- UNICEF. (2021). COVID-19 Vaccine Market Dashboard. Unicef.Org [blog], 22-7.2020. Disponible en: https://www.unicef.org/supply/covid-19-vaccine-market-dashboard

- Voituriez, T. y Chancel L. (2021). Developing countries in times of COVID: Comparing inequality impacts and policy responses. Paris: World Inequality Lab. Disponible en: https://wid.world/document/developing-countries-in-times-of-covid-comparing-inequality-impacts-and-policy-responses-world-inequality-lab-issue-brief-2021-01/

- Organización Mundial de la Salud. (2020). WHO Target Product Profiles for COVID-19 Vaccines (Publicación de la Organización Mundial de la Salud) . Ginebra: OMS. Disponible en: https://www.who.int/blueprint/priority-diseases/key-action/WHO_Target_Product_ Profiles_for_COVID-19_web.pdf

- Organización Mundial de la Salud. (2020). Sage Roadmap for prioritizing uses of Covid-19 vaccines in the context of limited supply (SAGE Working Group, Organización Mundial de la Salud). Ginebra: OMS. Disponible en: https://www.who.int/docs/default-source/ immunization/sage/covid/sage-prioritization-roadmap-covid19-vaccines.pdf?Status=Te mp\&sfvrsn=bf227443_2

- Organización Mundial de la Salud. ( 2020) . WHO SAGE values framework for the allocation and prioritization of COVID-19 vaccination (SAGE Working Group, Organización Mundial de la Salud). Ginebra: OMS. Disponible en: https://apps.who.int/iris/handle/10665/334299. 